\title{
Atypical choroid plexus papilloma: spontaneous resolution of diffuse leptomeningeal contrast enhancement after primary tumor removal in 2 pediatric cases
}

\author{
Marcello Scala, MD, ${ }^{1}$ Giovanni Morana, MD, PhD, ${ }^{2}$ Claudia Milanaccio, MD, ${ }^{1}$ Marco Pavanello, MD, ${ }^{3}$ \\ Paolo Nozza, MD, ${ }^{4}$ and Maria Luisa Garrè, MD ${ }^{1}$ \\ ${ }^{1}$ Neuro-Oncology Unit, ${ }^{2}$ Neuroradiology Unit, and Departments of ${ }^{3}$ Neurosurgery and ${ }^{4}$ Pathology, Istituto Giannina Gaslini, \\ Genova, Italy
}

Atypical choroid plexus papillomas can metastasize in the form of leptomeningeal seeding. Postoperative chemotherapy is the recommended first-line treatment when gross-total removal is not achieved or in cases of disseminated disease. Here the authors report on 2 children with atypical choroid plexus papillomas and MRI findings of diffuse leptomeningeal enhancement at diagnosis, later presenting with spontaneous resolution of the leptomeningeal involvement after removal of the primary lesions. Observations in this report expand our knowledge about the natural history and biological behavior of these tumors and highlight the role of close neuroimaging surveillance in the management of atypical choroid plexus papillomas in cases with MRI evidence of diffuse leptomeningeal enhancement at presentation.

https://thejns.org/doi/abs/10.3171/2017.2.PEDS16526

KEY WORDS brain tumor; pediatric; atypical choroid plexus papilloma; leptomeningeal dissemination; oncology

\footnotetext{
$\mathcal{O}^{2}$
} HOROID plexus tumors (CPTs) account for $1 \%-4 \%$ of all brain neoplasms in the pediatric age group and $13 \%$ of brain tumors occurring in the 1st year of life. ${ }^{14}$ On the basis of their histological features, these tumors are classified as choroid plexus papilloma (CPP; WHO Grade I), atypical CPP (ACPP; WHO Grade II), and choroid plexus carcinoma (CPC; WHO Grade III) according to the 2016 WHO classification..$^{0}$ Atypical CPP was recognized as a distinct entity in the 2007 WHO Classification of Tumours of the Central Nervous System, ${ }^{9}$ characterized by increased mitotic activity and a higher probability of recurrence as compared with CPP. The prognostic features of and clinical outcome rates for ACPP are between those displayed by CPP and CPC. Choroid plexus tumors can metastasize as solid nodules or as subarachnoid seeding, especially to the spine in patients with posterior fossa tumors. ${ }^{7}$ Metastases from CPP are rare and few cases have been reported. On the other hand, ACPP and CPC metastasize with greater frequency. ${ }^{17}$ Here we report 2 pediatric cases of ACPP presenting with diffuse leptomeningeal contrast enhancement on baseline MRI, which spontaneously resolved after gross-total removal of the primary tumor.

\section{Case Reports}

Case 1

History and Examination

An 8-month-old boy was admitted for the gradual onset of weight loss, poor feeding, downward gaze, and vomiting. At presentation, he was macrocephalic with bilateral papilledema. Brain MRI showed a huge cauliflower-like, contrast-enhancing mass filling and expanding the third ventricle (Fig. 1A), without evidence of parenchymal invasion. There was concomitant hydrocephalus. Diffuse pathological contrast enhancement was present in the basal

ABBREVIATIONS ACPP = atypical $\mathrm{CPP} ; \mathrm{CPC}=$ choroid plexus carcinoma; $\mathrm{CPP}=$ choroid plexus papilloma; $\mathrm{CPT}=$ choroid plexus tumor; $\mathrm{CSF}=$ cerebrospinal fluid . SUBMITTED February 11, 2017. ACCEPTED February 23, 2017. 

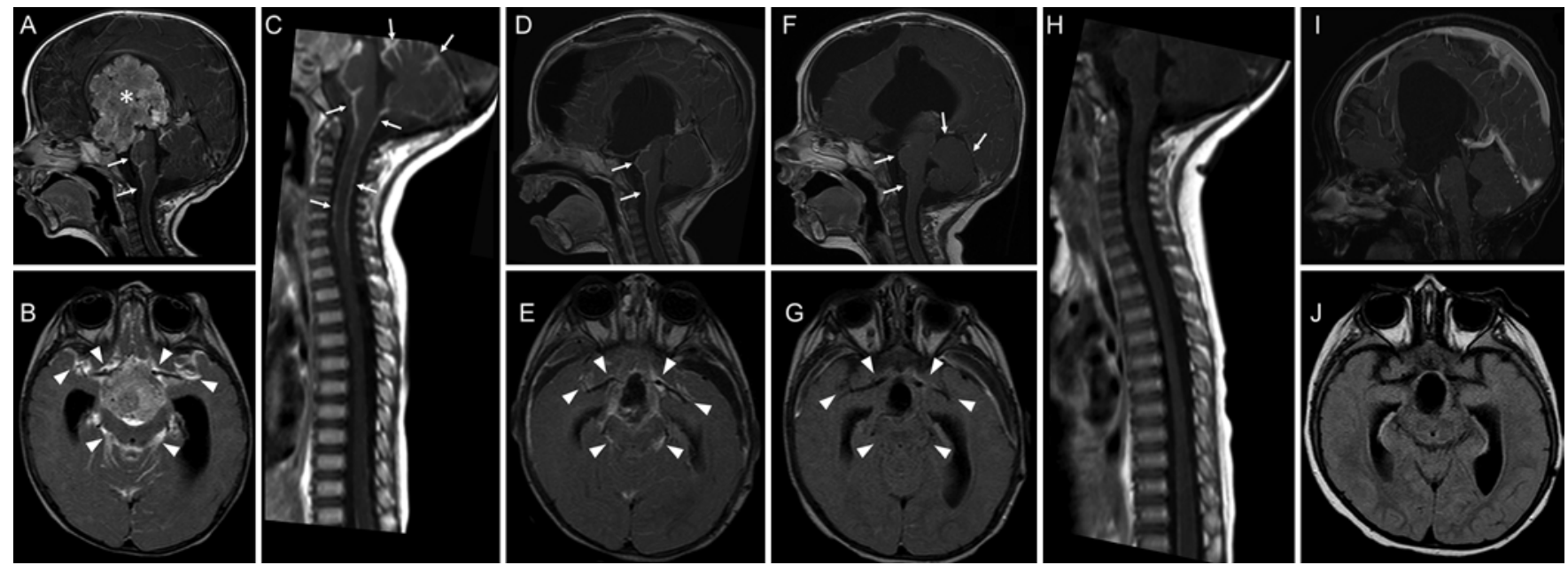

FIG. 1. Case 1. Admission sagittal Gd-enhanced T1-weighted MR image shows a huge, contrast-enhancing mass lesion filling the third ventricle (asterisk, A). There is concomitant pathological enhancement along the ventral profile of the brainstem (arrows) Axial Gd-enhanced FLAIR image demonstrates diffuse pathological enhancement in the basal cisterns (arrowheads, B). Sagittal Gd-enhanced T1-weighted MR image of the spine confirmed pathological leptomeningeal enhancement in the posterior cranial fossa and demonstrated linear enhancement along the anterior and posterior margins of the cervical spinal cord (arrows, C). Sagittal Gd-enhanced T1-weighted image obtained 1 week after the second surgery, showing complete removal of the mass and the persistence of leptomeningeal enhancement along the ventral profile of the brainstem (arrows, D). Axial Gd-enhanced FLAIR image shows diffuse reduction in arachnoidal enhancement in the basal cisterns (arrowheads, E). There are concomitant subdural collections. One-month follow-up sagittal Gd-enhanced T1-weighted MR (F) and axial postcontrast FLAIR (G) images of the brain show almost complete resolution of the previously seen leptomeningeal enhancement (arrows and arrowheads, respectively). Subdural collections are still present. Sagittal Gd-enhanced T1-weighted MR image $(\mathbf{H})$ of the spine demonstrates that the leptomeningeal enhancement along the cervical spinal cord is no longer visible. Twenty-six-month follow-up sagittal Gd-enhanced T1-weighted MR (I) and axial postcontrast FLAIR (J) images show neither evidence of local disease nor signs of secondary dissemination with complete reabsorption of the subdural collections.

cisterns, on the surface of both cerebral hemispheres, and along the spinal cord (Fig. 1A-C).

\section{Operation}

The tumor was surgically treated via interhemispheric transcallosal approach. However, because of intraoperative bleeding, tumor removal was limited to a partial resection. Both lumbar and ventricular cerebrospinal fluid (CSF) collections were negative for neoplastic cells.

\section{Pathological Findings}

Neuropathological examination showed a papillary CPT with large areas of necrosis, without nuclear pleomorphism. Three mitoses per 10 randomly selected high-power fields were found. The Ki-67 proliferation index was 9\%. Brain invasion was not found. The diagnosis was ACPP.

\section{Second Operation and Postoperative Course}

Two weeks after the initial resection, residual tumor was completely removed via a left frontal transcortical approach. One week later, postoperative MRI was performed as staging before the start of chemotherapy, which was considered given the presence of leptomeningeal enhancement on the preoperative scan. Magnetic resonance imaging confirmed gross-total removal and showed a reduction in leptomeningeal intracranial involvement (Fig. 1D and E). Strict MRI follow-up was then planned and no chemotherapy treatment was adopted. One month later,
MRI demonstrated almost complete resolution of the intracranial and spinal leptomeningeal enhancement (Fig. $1 \mathrm{~F}-\mathrm{H})$, which was no longer visible on a subsequent MRI performed 5 months later. The patient is still disease free 26 months after surgery (Fig. 1I and J).

\section{Case 2}

History and Examination

An 11-month-old boy was referred to our hospital because of macrocephaly. Neurological examination and funduscopy were normal. Brain MRI showed a large, left intraventricular, polylobulated contrast-enhancing mass originating from the choroid glomus and extending into the ipsilateral temporal and occipital horns (Fig. 2A-C). No parenchymal infiltration was evident. Diffuse leptomeningeal enhancement was demonstrated into the basal cisterns and along the brainstem.

\section{Operation}

A left temporoparietal craniectomy was performed and gross-total tumor removal was achieved. Cerebrospinal fluid collections from lumbar puncture and ventricles were negative.

\section{Pathological Findings}

Neuropathological examination showed a papillary CPT with small areas of necrosis, without nuclear pleomorphism. Three mitoses per 10 randomly selected highpower fields were found. The Ki-67 proliferation index was $7 \%$. Brain invasion was not found. 

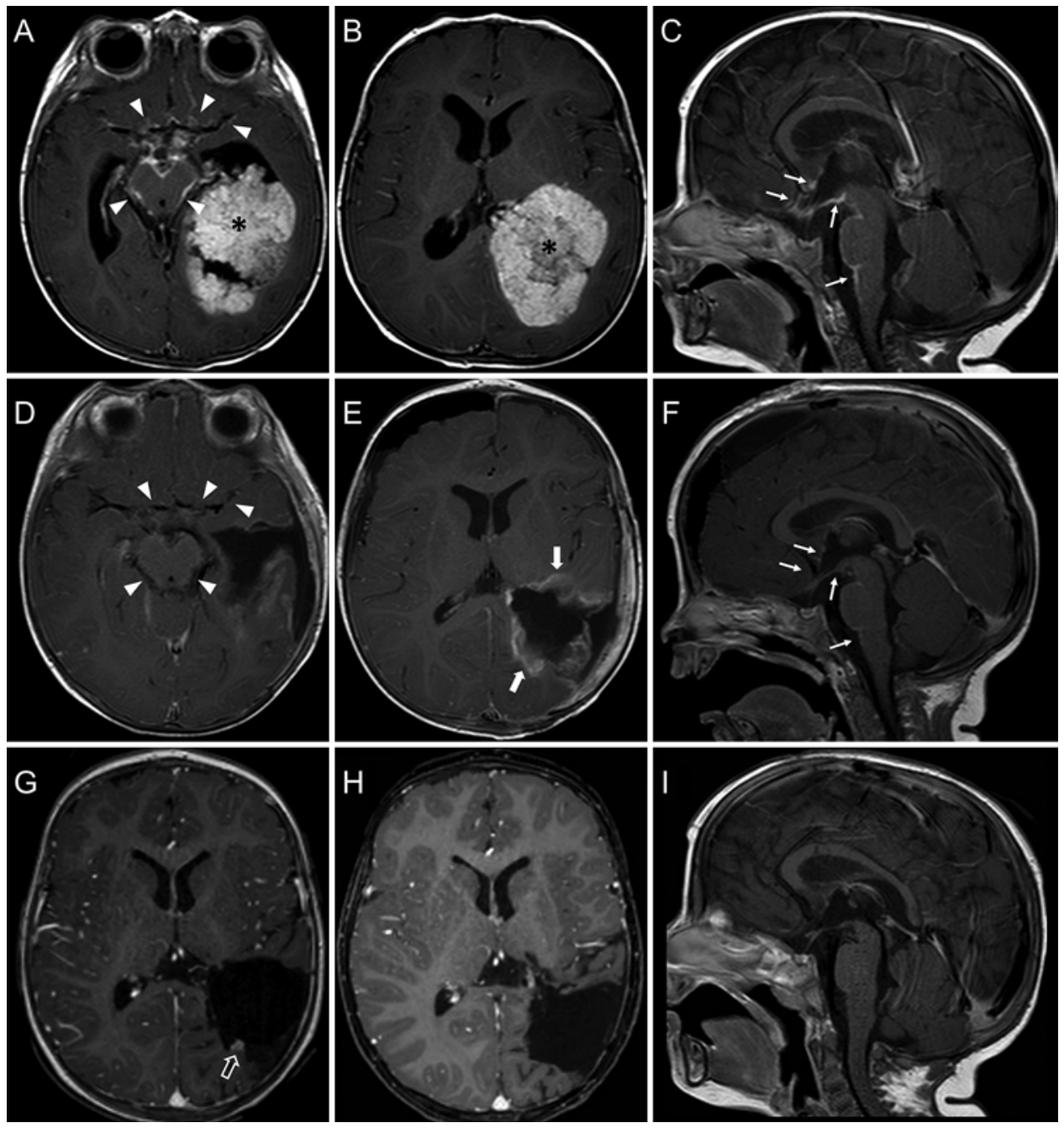

FIG. 2. Case 2. Admission axial Gd-enhanced T1-weighted MR images show a large, intraventricular, contrast-enhancing mass lesion with its epicenter in the left atrium and extending into the ipsilateral occipital and temporal horns (asterisks, A and B). There is also pathological enhancement in the basal cisterns (arrowheads, A). Midline sagittal Gd-enhanced T1-weighted MR image confirms this finding and shows pial enhancement along the ventral surface of the brainstem (thin arrows, C). Three-week postoperative axial ( $\mathbf{D}$ and $\mathbf{E}$ ) and sagittal $(\mathbf{F}) \mathrm{Gd}$-enhanced T1-weighted MR images show removal of the mass lesion and almost complete resolution of leptomeningeal enhancement (arrowheads, D; thin arrows, F). Reactive postsurgical linear enhancement is visible along the margins of the surgical cavity (thick arrows, E). Twelve-month postoperative axial Gd-enhanced T1-weighted MR image shows a small nodular lesion along the posterior margin of the surgical cavity (open arrow, $\mathbf{G}$ ), in keeping with a local relapse (likely growth of a microresidual component). There is no evidence of leptomeningeal enhancement. Forty-three-month axial $(\mathbf{H})$ and sagittal (I) Gd-enhanced T1-weighted MR images show no evidence of local and distant disease.

\section{Postoperative Course}

Magnetic resonance imaging performed 3 weeks after surgery (Fig. 2D-F) did not show residual disease and demonstrated also almost complete resolution of the leptomeningeal enhancement, which was no longer visible on subsequent MRI performed 1 month later. However, surveillance neuroimaging performed at 12 months revealed a small, growing, contrast-enhancing lesion located along the posterior margin of the surgical cavity (Fig. 2G). The nodule was surgically removed. No neoplastic cells were found in the ventricular CSF samples. The diagnosis of ACPP was confirmed. Two months later, MRI showed no residual disease and no signs of leptomeningeal involvement. Chemotherapy was never administered and the pa- tient is still disease free 43 months after the first surgery (Fig. 2H and I).

\section{Discussion}

Choroid plexus papillomas metastasize as intraparenchymal or intraventricular nodules, especially subarachnoid nodules, or less frequently as diffuse leptomeningeal seeding. ${ }^{1,2,4-6,8,12,13,15}$ Metastases at diagnosis in cases of ACPP are not uncommon and have been reported in 17\% of cases according to the Choroid Plexus Tumor-Society of Pediatric Oncology-2000 (CPT-SIOP-2000) study of CPTs. ${ }^{17}$ In cases of metastases, local recurrence, or incomplete resection, chemotherapy is recommended and the pa- 
tient should be evaluated for a second-look surgery thereafter. ${ }^{3,11}$ Radiation therapy has also been used in combination with chemotherapy in metastatic ACPP patients. ${ }^{3,11}$ Cases in the present report demonstrated diffuse, pathological leptomeningeal contrast enhancement on baseline MRI, strongly suspicious for arachnoidal infiltration and seeding, progressively and completely vanishing after surgical removal of the primary tumor. Similar observations have been reported in the literature but they involved adults with CPP.,16 To our knowledge, this phenomenon has never been described in ACPP and in children.

In our cases, the interval between surgery and complete disappearance of the leptomeningeal enhancement was a few months, with rapid evidence of a reduction in contrast enhancement on the earlier postoperative MRI studies performed 1 week after the second surgery in the first case and 3 weeks after surgery in the second case. This decrease in enhancement was the rationale behind the decision to adopt a "wait and see" approach with close neuroimaging and clinical monitoring and to avoid adjuvant chemotherapy. In the second child, a local recurrence occurred probably as a result of the growth of a microresidual component along the resection margins, without any MRI sign of arachnoidal involvement. After surgical removal of the lesion, the same follow-up strategy was adopted and no adjuvant therapy was started. No signs of local recurrence or dissemination appeared in subsequent MRI.

Regarding the nature of the leptomeningeal enhancement, it has been supposed that it could be the result of abnormal arachnoidal vessel permeability triggered by hormonal factors (such as vascular endothelial growth factor) secreted by the tumor or of real arachnoidal infiltration by a lining of neoplastic cells, depending on a critical concentration of growth factors secreted by the tumor. ${ }^{6,16}$ The removal of primary tumor and thus the lack of a triggering factor may explain the resolution of leptomeningeal enhancement. At the same time, we recognize that leptomeningeal enhancement does not necessarily mean malignant seeding since it has been described in cases of obstructive hydrocephalus (venous stasis) or of leptomeningeal irritation from postoperative subarachnoid bleeding in children with brain tumors. ${ }^{16}$ The latter does not apply in our patients since leptomeningeal enhancement was demonstrated before any surgical procedure.

Regardless of the true cause, MRI evaluation is standard in the determination of secondary dissemination in brain malignancies, and the neuro-oncological strategy depends on MRI data even without histological confirmation. In our cases, given the location of the leptomeningeal involvement (basal cisterns and spine), an additional surgical procedure would have been necessary to evaluate the nature of the contrast enhancement, exposing the children to additional risks; therefore, close MRI follow-up was the preferred strategy. Since postoperative chemotherapy is the recommended first-line treatment for ACPP in cases of disseminated disease, MRI evidence of diffuse leptomeningeal contrast enhancement at presentation in ACPP cases should be interpreted with caution, and restaging should be performed after gross-total removal or subtotal removal of the tumor. Indeed, leptomeningeal enhance- ment in the setting of ACPP may represent a tumor-related effect rather than true tumor dissemination. In conclusion, our observations expand our knowledge about the natural history and biological behavior of ACPP and highlight the role of close clinical and neuroimaging monitoring in the management of ACPP with MRI evidence of leptomeningeal involvement at presentation.

\section{Acknowledgments}

We acknowledge support from the Association for Pediatric Brain Tumors (Associazione per la ricerca sui tumori cerebrali del bambino) and the Berlucchi Foundation.

\section{References}

1. Enomoto H, Mizuno M, Katsumata T, Doi T: Intracranial metastasis of a choroid plexus papilloma originating in the cerebellopontine angle region: a case report. Surg Neurol 36:54-58, 1991

2. Guidetti B, Spallone A: The surgical treatment of choroid plexus papillomas: the results of 27 years experience. Neurosurg Rev 4:129-137, 1981

3. Heese O, Lamszus K, Grzyska U, Westphal M: Diffuse arachnoidal enhancement of a well differentiated choroid plexus papilloma. Acta Neurochir (Wien) 144:723-728, 2002

4. Jagielski J, Zabek M, Wierzba-Bobrowicz T, Łakomiec B, Chrapusta SJ: Disseminating histologically benign multiple papilloma of the choroid plexus: case report. Folia Neuropathol 39:209-213, 2001

5. Japp AS, Gessi M, Messing-Jünger M, Denkhaus D, Zur Mühlen A, Wolff JE, et al: High-resolution genomic analysis does not qualify atypical plexus papilloma as a separate entity among choroid plexus tumors. J Neuropathol Exp Neurol 74:110-120, 2015

6. Jinhu Y, Jianping D, Jun M, Hui S, Yepeng F: Metastasis of a histologically benign choroid plexus papilloma: case report and review of the literature. J Neurooncol 83:47-52, 2007

7. Leblanc R, Bekhor S, Melanson D, Carpenter S: Diffuse craniospinal seeding from a benign fourth ventricle choroid plexus papilloma. Case report. J Neurosurg 88:757-760, 1998

8. Leys D, Pasquier F, Lejeune JP, Lesoin F, Petit H, Delandsheer JM: [Benign choroid plexus papilloma. 2 local recurrences and intraventricular seeding.] Neurochirurgie 32:258-261, $1986(\mathrm{Fr})$

9. Louis DN, Ohgaki H, Wiestler OD, Cavenee WK, Burger PC, Jouvet A, et al: The 2007 WHO classification of tumours of the central nervous system. Acta Neuropathol 114:97-109, 2007

10. Louis DN, Perry A, Reifenberger G, von Deimling A, Figarella-Branger D, Cavenee WK, et al: The 2016 World Health Organization Classification of Tumors of the Central Nervous System: a summary. Acta Neuropathol 131:803-820, 2016

11. McCall T, Binning M, Blumenthal DT, Jensen RL: Variations of disseminated choroid plexus papilloma: 2 case reports and a review of the literature. Surg Neurol 66:62-68, 2006

12. Ortega-Martínez M, Cabezudo-Artero JM, FernándezPortales I, Pimentel JJ, Gómez de Tejada R: Diffuse leptomeningeal seeding from benign choroid plexus papilloma. Acta Neurochir (Wien) 149:1229-1237, 2007

13. Passariello A, Tufano M, Spennato P, Quaglietta L, Verrico A, Migliorati R, et al: The role of chemotherapy and surgical removal in the treatment of choroid plexus carcinomas and atypical papillomas. Childs Nerv Syst 31:1079-1088, 2015 
14. Rickert $\mathrm{CH}$, Paulus W: Tumors of the choroid plexus. Microsc Res Tech 52:104-111, 2001

15. Shakespeare TP, Slancar MM, Mallik AR, Bell DR: CSF dissemination of a benign choroid plexus papilloma (CPP). Aust N Z J Med 27:597-598, 1997

16. Wiener MD, Boyko OB, Friedman HS, Hockenberger B, Oakes WJ: False-positive spinal MR findings for subarachnoid spread of primary CNS tumor in postoperative pediatric patients. AJNR Am J Neuroradiol 11:1100-1103, 1990

17. Wrede B, Hasselblatt M, Peters O, Thall PF, Kutluk T, Moghrabi A, et al: Atypical choroid plexus papilloma: clinical experience in the CPT-SIOP-2000 study. J Neurooncol 95:383-392, 2009

\section{Disclosures}

The authors report no conflict of interest concerning the materi- als or methods used in this study or the findings specified in this paper.

\section{Author Contributions}

Conception and design: Scala, Morana, Garrè. Acquisition of data: Scala, Morana. Analysis and interpretation of data: Scala, Morana, Garrè. Drafting the article: Scala, Morana. Critically revising the article: all authors. Reviewed submitted version of manuscript: all authors. Approved the final version of the manuscript on behalf of all authors: Scala. Study supervision: Morana, Garrè.

\section{Correspondence}

Marcello Scala, Neuro-Oncology Unit, Istituto Giannina Gaslini, Via Gerolamo Gaslini, 5, Genova 16148, Italy. email: marcelloscala87@gmail.com. 